\title{
MONODROMY AND THE BOHR-SOMMERFELD GEOMETRIC QUANTIZATION
}

\author{
NICOLA SANSONETTO
}

Communicated by Jan J. Sławianowski

Abstract. We study the linear part of the monodromy of completely integrable Hamiltonian systems via Bohr-Sommerfeld Geometric Quantization. We relate monodromy to the ambiguity in the choice of the pre-quantum connection and to the action of the (connected component of the) gauge group.

\section{Introduction}

In the framework of Bohr-Sommerfeld geometric quantization, we study (quantum) monodromy from different viewpoints. Monodromy, together with the socalled Chern-Duistermaat class and the Lagrangian class, provides an obstruction to the global definition of action-angle variables for completely integrable Hamiltonian systems $[7,9]$. Our specific contributions relate monodromy to the freedom of choice of a pre-quantum connection and to $\mathcal{G}_{0}$-equivalence of connections $\left(\mathcal{G}_{0}\right.$ is the connected component of the identity of the gauge group $\mathcal{G}$ of a pre-quantum line bundle).

The present work is organized as follows. In Section 2 we first review LiouvilleArnold theorem and the obstructions to existence of global action-angles coordinates and then we quickly review the geometric quantization method. In Section 3 we state and prove the main results of the paper. A short section with conclusions and perspectives follows.

\section{Liouville-Arnold Theorem and Geometric Quantization}

In this section we review some basic facts about completely integrable Hamiltonian systems and geometric quantization. We will also introduce the notation that will be used throughout the paper. 


\subsection{Completely Integrable Hamiltonian Systems}

Let $(M, \omega)$ be a $2 n$-dimensional symplectic manifold, and fix $h: M \longrightarrow \mathbb{R}$, a smooth function on $M$ (the Hamiltonian), with its associated vector field $X_{h}$, fulfilling $i_{X_{h}} \omega=-\mathrm{d} h$. The triple $(M, \omega, h)$ is called a Hamiltonian system on $M$, and it is said to be completely integrable in a subset $\widetilde{M}$ of $M$ if it admits $n$ mutually Poisson-commuting first integrals, which are functionally independent almost everywhere in $M$, and, restricting the latter, if necessary, the joint level sets of the first integrals are compact and connected. The Liouville-Arnold Theorem (see e.g. $[1,7]$ ) gives sufficient conditions for the complete integrability of a Hamiltonian system.

Theorem 1 (Liouville-Arnold). Let $(M, \omega)$ be a $2 n$-dimensional symplectic manifold. Let $f=\left(f_{1}, \ldots, f_{n}\right): M \longrightarrow \mathbb{R}^{n}$

- be a surjective submersion (i.e., the energy-momentum mapping)

- have compact and connected fibers $f^{-1}(x)$

- its components pairwise Poisson-commute, i.e., $\left\{f_{i}, f_{j}\right\}=0$ for every $i, j=$ $1, \ldots, n$.

Let $A$ be the set of regular values of $f$. Then for each $x \in A$

1. the fibers $f^{-1}(x)$ of $f$ are diffeomorphic to $\mathbb{T}^{n}$

2. there exists an open neighborhood $U_{x}$ of $x$ in $A$ and a diffeomorphism $(\boldsymbol{a}, \boldsymbol{\alpha}): f^{-1}\left(U_{x}\right) \longrightarrow V \times \mathbb{T}^{n}$ with $V$ an open subset of $\mathbb{R}^{n}$ such that $\boldsymbol{a}=\left(a_{1}, \cdots, a_{n}\right)=\kappa \circ f$ for some diffeomorphism $\kappa: f\left(U_{x}\right) \longrightarrow V$

3. the coordinates $(\boldsymbol{a}, \boldsymbol{\alpha})$ on $M$ are Darboux coordinates, the so-called actionangles coordinates, that is $\omega=\mathrm{d} \boldsymbol{a} \wedge \mathrm{d} \boldsymbol{\alpha}$.

From the geometric point of view the Liouville-Arnold Theorem ensures that $M$ has a $\mathbb{T}^{n}$-bundle structure with Lagrangian fibers and moreover, $f^{-1}(A)$ is a local toric principal bundle with structure group $\mathbb{T}^{n}$ with Lagrangian fibers, whose structure group $\mathbb{T}^{n}$ acts in a Hamiltonian way, with momentum map given by the projection bundle map. Besides the action-angles coordinates are bundle coordinates. We want to stress that the construction of the toric principal bundle or, equivalently, the existence of global action-angle coordinates is only local. LiouvilleArnold Theorem also implies that the base manifold $A$ of the $\mathbb{T}^{n}$-bundle is an integral affine manifold. Since also the fibers of the bundle carry an affine structure, the 
transition functions between two intersecting domain of action-angle coordinates $(\boldsymbol{a}, \boldsymbol{\alpha})$ and $\left(\boldsymbol{a}^{\prime}, \boldsymbol{\alpha}^{\prime}\right)$ are

$$
\boldsymbol{a}^{\prime}=Z^{-T} \boldsymbol{a}+\boldsymbol{z}, \quad \boldsymbol{\alpha}^{\prime}=Z \boldsymbol{\alpha}+\mathcal{F}(\boldsymbol{a}) \quad \bmod 2 \pi
$$

where $Z \in \mathrm{SL}_{n}(\mathbb{Z}), \boldsymbol{z} \in \mathbb{R}^{n}$ and $\mathcal{F}: a\left(\pi^{-1}(V) \cap \pi^{-1}\left(V^{\prime}\right)\right) \longrightarrow \mathbb{R}^{n}$ such that $\partial_{a_{i}}(Z \mathcal{F})_{j}=\partial_{a_{j}}(Z \mathcal{F})_{i}$ with $i, j=1, \ldots, n$. From (1) clearly follows the non-uniqueness of the action-angle coordinates which is due to the three arbitrary choices that must be made in the proof of Liouville-Arnold Theorem - first, the choice of a basis of the period lattice, second a choice of a local Lagrangian section (that is an origin to count the angles) and finally a constant of the integration in the derivation of the actions. This freedom affects the globalization of the construction of Liouville-Arnold Theorem. A first answer was given in terms of cocycles by Nekhoroshev [9] in 1976 and exhaustively and independently by Duistermaat [7] in 1980 , in terms of the sheaf theory.

Theorem 2 ([7]). With the notation of the previous theorem, the torus bundle $\pi$ : $f^{-1}(A) \longrightarrow \mathbb{R}^{n}$ is topologically trivial if and only if the monodromy and the Chern-Duistermaat class of the $\mathbb{T}^{n}$-bundle are trivial. Moreover if the symplectic form is exact then the existence of global action-angle coordinates is equivalent to the triviality of the Lagrangian toric fibration.

Remarks 3. - The Chern-Duistermaat class is the Chern class of the bundle and it describes the obstruction to the existence of a global section of the bundle (we referee to [2,12] for a detailed discussion on the Chern class in the case of completely integrable Hamiltonian systems). In local terms it means that even if the action variables are globally defined, the function $\mathcal{F}$ in (1) is not.

- In the case of a system with two degrees of freedom possessing an isolated critical value (of focus-focus type) of the energy-momentum map $f$, the Chern-Duistermaat class is trivial since the base manifold A admits a Leray cover with empty triple intersections. Therefore the only obstruction to the triviality of the fibration is the monodromy.

The coarsest and most known obstruction to the global existence of the actionangles coordinates is the monodromy (actually its linear part), which is the one we are interested in this paper. From the geometric point of view the monodromy is the obstruction to the global "principality" of the toric bundle. Equivalently, taken a point $x$ in $A$ and a basis for the first homology group of the fiber $\pi^{-1}(x)$ over $x$, if we carry it over loops in $A$, when we arrive again back at $x$, we have a map 
that describes the change of the basis of the first homology of the fibers and it depends only on the homotopy type of $A$. Therefore the monodromy is given by the representation (Duistermaat's idea)

$$
\mathcal{M}: \pi_{1}(A) \longrightarrow \operatorname{Aut}\left(\mathrm{H}_{1}\left(\mathbb{T}^{n}, \mathbb{Z}\right)\right) \cong \mathrm{SL}_{n}(\mathbb{Z})
$$

From a local point of view, (Nekhoroshev's idea) the monodromy is the obstruction to patch together charts on the base manifold $A$ around the singularities. Indeed the product of $r>1$ matrices in $\mathrm{SL}_{n}(\mathbb{Z})$ need not to be the identity. Another characterization of monodromy has been suggested by Weinstein [7], but see [2] for a detailed discussion in terms of the holonomy of a suitable flat connection. Since every Lagrangian fibration admits an affine, flat and torsion free connection induced by the standard connection of $\mathbb{R}^{n}$ (see [13]), it turns out that the holonomy of this connection is the monodromy of the bundle. Let us discuss more in detail this aspect since it will be crucial for the sequel. It will be convenient for us to study Hamiltonian monodromy from a differential geometric point of view (see $[5,7,13]$ ). Indeed, it is well-known (see [13]) that a Lagrangian fibration admits an affine, flat, torsion free connection $\nabla^{\mathrm{Ehr}}: T M \longrightarrow V M$ (the vertical bundle over $M$ ) on the Lagrangian leaves, which is an Ehresmann good connection for the fibration (i.e., that is every smooth curve on the base has a horizontal lift). The $\mathrm{GL}(n, \mathbb{Z})$-holonomy representation $\mathrm{hol}\left(\nabla^{\mathrm{Ehr}}\right)$ of $\nabla^{\mathrm{Ehr}}$ is the monodromy representation $\mathcal{M}\left(\pi_{1}(A)\right)$ of the $\mathbb{T}^{n}$-bundle over $A$. Moreover the monodromy representation actually takes values in $\operatorname{SL}(n, \mathbb{Z})$ upon choosing suitable bases of the tangent spaces of the base space.

Remarks 4. - In [8] (and independently in [15]) is given a sufficient condition for the non-triviality of monodromy near isolated focus-focus singularities: more precisely, the (local) monodromy near a topologically stable focusfocus point (in the interior of the energy-momentum range) is non-trivial.

- There are various examples of completely integrable Hamiltonian systems that present monodromy: the spherical pendulum [5,7], the champagne bottle [2], the Lagrange top (see [5] and reference therein).

- Upon quantization of a completely integrable Hamiltonian system, one has a natural notion of quantum monodromy $\mu_{q}$ which is equal to $\left(\mu_{c}\right)^{-T}$, where $\mu_{c}$ denotes the classical monodromy. (See [10] for a rigorous introduction to quantum monodromy). 


\subsection{Geometric Quantization}

Let us now briefly review the basics of geometric quantization. For a complete account we refer to [3,14]. Recall that if $(M, \omega)$ is a real symplectic manifold of even dimension such that $\left[\frac{1}{2 \pi} \omega\right] \in \mathrm{H}^{2}(M, \mathbb{Z})$, then the Weil-Kostant Theorem states that there exists a complex line bundle $(L, \nabla, h)$ over $M$ equipped with a hermitian metric $h$ and a compatible connection $\nabla$ with curvature $F_{\nabla}=\omega$. Hence $[\omega]=c_{1}(L)$, the first Chern class of $L \rightarrow M$. The connection $\nabla$ is called a pre-quantum connection and $L \rightarrow M$ the pre-quantum line bundle. The different choices of $L \rightarrow M$ and $\nabla$ are parametrized by the first cohomology group $\mathrm{H}^{1}\left(M, \mathbb{S}^{1}\right)$ (see e.g. [14]). In more detail given any complex line bundle $L \rightarrow M$, the connections thereon are classified, up to gauge equivalence, by their curvature (fixing the topological type of the line bundle, via the first Chern class) and by their holonomy, specified, in turn, on a basis of (real) homology one-cycles $\left[\gamma_{i}\right]$, for $\mathrm{H}_{1}(M, \mathbb{R})$, of dimension $b_{1}$, the first Betti number of $M$ - represented, for instance, by smooth curves passing through a given point. The holonomy is trivial if $M$ is simply connected. The gauge group $\mathcal{G}$ consists, in this case, of all smooth maps $g: M \rightarrow \mathbb{S}^{1}$ - explicitly, $g: x \mapsto \mathrm{e}^{\mathrm{i} \varphi(x)}$, obvious notation - and it is not connected in general, its connected components being parametrized by the degree of the maps $g: M \rightarrow \mathbb{S}^{1}$. The connected component (of the identity) of $\mathcal{G}$ will be denoted by $\mathcal{G}_{0}$, as usual, and will play an important role in what follows.

Given a connection $\nabla_{0}$, any other connection is of the form $\nabla=\nabla_{0}+a$, with $a \in \Lambda^{1}(M)$, (i.e., they build up an affine space modelled on the space of one-forms $\left.\Lambda^{1}(M)\right)$ and the relation between their respective curvatures is $F_{\nabla}=F_{\nabla_{0}}+\mathrm{d} \eta$ Therefore, the curvatures are the same if and only if $\eta$ is closed. This being the case, $a$ determines a de Rham cohomology class $[a] \in \mathrm{H}^{1}(M, \mathbb{R})$, fully recovered via the period map

$$
\mathrm{H}^{1}(M, \mathbb{R}) \ni[a] \mapsto\left(\int_{\gamma_{1}} a, \ldots, \int_{\gamma_{b_{1}}} a\right) \in \mathbb{R}^{n} .
$$

The gauge group $\mathcal{G}$ acts on connections via $\nabla \mapsto \nabla+g \cdot \mathrm{d} g^{-1}=\nabla-\mathrm{i} \mathrm{d} \varphi$. Therefore, the set of all gauge inequivalent connections (possessing the same curvature) is clearly given by $\mathrm{H}^{1}(M, \mathbb{R}) / \mathrm{H}^{1}(M, \mathbb{Z})$ and, if $M$ is a torus, then the above set is again a torus, the Jacobian of $M$. If the initial connection has zero curvature, then the above space parametrises flat connections up to gauge equivalence.

Coming back to the specific geometric quantization setting, given a Lagrangian submanifold $\Lambda$ of the symplectic manifold $M$, the symplectic two-form $\omega$ vanishes upon restriction to $\Lambda$ by definition, and any (semi-local) symplectic potential 
$\theta$ becomes a closed form thereon, defining a (semi-local) connection form pertaining to the restriction of the pre-quantum connection $\nabla$, denoted by the same symbol. The latter is a flat connection and a global covariantly constant section of the restriction of the pre-quantum line bundle exists if and only if it has trivial holonomy, that is the Bohr-Sommerfeld condition is fulfilled

$$
\left[\frac{1}{2 \pi} \theta\right] \in \mathrm{H}^{1}(M, \mathbb{Z}) \quad \text { i.e., } \quad \int_{\gamma} \theta \in 2 \pi \mathbb{Z}
$$

for any closed loop $\gamma$ in $\Lambda$.

A covariantly constant section (which we call WKB-, or BS-wave function) takes the form

$$
s(m):=\operatorname{hol}_{\gamma}(\nabla) \cdot s\left(m_{0}\right)=\mathrm{e}^{\mathrm{i} \int_{\gamma} \theta} s\left(m_{0}\right)
$$

with $\gamma$ denoting any path connecting a chosen point $m_{0}$ in $\Lambda$ with a generic point $m \in \Lambda$, $\operatorname{hol}_{\gamma}(\nabla)$ being the holonomy along $\gamma$ of the restriction to $\Lambda$ of the prequantum connection $\nabla$. The r.h.s. of (4) tacitly assumes the choice of a trivialization of $\left.L\right|_{\Lambda} \longrightarrow \Lambda$ around $m_{0}$ and $m$ in a corresponding local chart.

Remarks 5. 1. We stress the fact that the Bohr-Sommerfeld condition forces us to deal with $\mathcal{G}_{0}$-equivalence classes (i.e., the degree of the gauge maps must be zero) in order to avoid trivialities.

2. Our definition of $W K B$-wave function is slightly different from the conventional one (see e.g. [14]). Indeed we do not require square-integrability and we do not twist the prequantization bundle with $\Delta_{\nabla}$ (whose smooth sections consist of the complex $n$-forms on $\Lambda$ ), thus neglecting the "amplitudesquared".

3. There is a version of the Bohr-Sommerfeld condition incorporating the Maslov class, but we shall not need this refinement in what follows.

We also recall that the pre-quantum connection $\nabla$ allows the construction of the (Hermitian) pre-quantum observables $Q(\cdot)$ via the formula

$$
Q(f)=-\mathrm{i} \nabla_{X_{f}}+f=-\mathrm{i}\left(X_{f}-i_{X_{f}} \theta\right)+f
$$

with $f$ a smooth function on $M$. The connection is determined up to a closed one-form, yielding a corresponding ambiguity in the definition of the quantum observable $Q(f)$ attached to $f$. This fact will be exploited in the sequel. 


\section{Monodromy via Bohr-Sommerfeld Geometric Quantization}

In this section we will detect (quantum) monodromy via Bohr-Sommerfeld geometric quantization and analyse how the monodromy itself affects geometric quantization as well in different ways.

Let us consider the geometric quantization of a completely integrable Hamiltonian system on a symplectic manifold of dimension $2 n$ with vanishing Chern class and with vanishing affine monodromy, i.e. the vector $z$ and the function $\mathcal{F}$ in (1) must vanish. (This two assumptions are not necessary for the result of our work but will simplify the exposition and improve the clarity of the results).

\subsection{The Pre-quantum Connection}

In this section we show that monodromy can be detected exploiting the freedom in the choice of the pre-quantum connection.

Let us perform geometric quantization in a neighborhood $U$ of a Lagrangian torus $\mathbb{T}^{n}$. Let $L$ denote the pre-quantum line bundle and $\nabla$ the pre-quantum connection. Moreover let $\theta$ be the (local) connection form.

\section{Theorem 6 ([11]).}

A. The monodromy is the holonomy of the so-called $\mathrm{BS}$-adapted connection induced by the Liouville one-form.

B. The monodromy is the holonomy of the so-called monodromy connection induced by the vertical one-form, which in coordinates reads $\theta^{\prime}=-\boldsymbol{\alpha} \mathrm{d} \boldsymbol{a}$.

\section{Proof:}

A. Consider the standard connection $\nabla$ given by the Liouville one-form $\theta=$ $\boldsymbol{a} \mathrm{d} \boldsymbol{\alpha}$. Since this connection is vertical we call it a BS-adapted connection. It fulfills $\nabla_{X_{b}}=X_{b}$ with $X_{a}$ any vector field tangent to a Lagrangian section. Moreover it is obviously flat along the fibers, since the restriction of the symplectic form on the fibers vanishes, being the fibers Lagrangian submanifolds. Given a BS-adapted connection, the action variables may be recover as follow $a_{k}=\frac{1}{2 \pi i} \log \operatorname{hol}\left(\left.\nabla\right|_{\mathbb{T}^{n}}, \gamma_{k}\right)$ where $\gamma_{k}$ 's yield a basis of one-cycles in $\mathbb{T}^{n}$, thus making clear local definition of the actions. Hence, monodromy may be view as the obstruction to patch together geometric quantization bundles equipped with a local BS-adapted connection. Note, however, that there is non global obstruction to prequantization by the Weil-Kostant Theorem. 
B. Consider now the connection $\nabla^{\prime}$ defined by the form $\theta^{\prime}=-\boldsymbol{\alpha} \mathrm{d} \boldsymbol{a}$. We call this connection a monodromy connection, since parallel transport along a non trivial loop contained in a local Lagrangian section $\alpha=c$, whereupon it is flat, produces a holonomy hol $\left(\nabla^{\prime}\right)=\mathrm{e}^{-\mathrm{i} \Delta \boldsymbol{a}}$ due to the possible nonglobality of the action variables.

\subsection{The Gauge Approach}

In this section show how to detect monodromy using a gauge-equivalence theoretic interpretation of the pre-quantum connections.

\section{Theorem 7 ([11]).}

1. The monodromy representation (2) can be viewed as a map $\widetilde{M}: \pi_{1}(A) \longrightarrow$ $\mathcal{G} / \mathcal{G}_{0} \cong \mathrm{SL}_{n}(\mathbb{Z})$, which acts transitively on $\mathcal{B} S$, as expression (5) below shows, and can be read both on wave functions and observables.

2. Take a BS-adapted connection and perform a change of coordinates according with (1), then, remaining in the same Hilbert space, monodromy eventually induces a change in the quantum action operator.

\section{Proof:}

1. Upon enforcing Bohr-Sommerfeld condition take the integral de Rham class of $\nabla:=\left.\nabla\right|_{\mathbb{T}^{n}}$. i.e., $[\theta]$ via the period map (3), and denote by $\mathcal{B S}$ the set of all classes $\left[\theta_{\nabla}\right]$. Then

$$
\mathcal{B S} \cong \mathrm{H}^{1}\left(\mathbb{T}^{n}, \mathbb{Z}\right)=\mathcal{G} \cdot\left[\nabla_{0}\right]
$$

with $\nabla_{0}$ a fixed flat connection. Thus $\mathcal{B S}$ is a $\mathcal{G}$-homogeneous space isomorphic to $\mathbb{Z}^{n}$, whereupon the connected component $\mathcal{G}_{0}$ of the gauge group $\mathcal{G}$ acts trivially. Hence $\mathcal{G} / \mathcal{G}_{0} \cong \mathrm{SL}_{n}(\mathbb{Z})$ acts freely on $\mathcal{B S}$ and provides a natural (quantum) monodromy representation: $\widetilde{\mathcal{M}}: \pi_{1}(A) \longrightarrow \mathcal{G} / \mathcal{G}_{0} \cong$ $\mathrm{SL}_{n}\left(\mathbb{Z}^{n}\right)$.

2. Let us perform a Darboux change of coordinates on a fixed BS-torus according to (1). Then extend the change of coordinates to a canonical transformation in a neighborhood of the fixed torus: $\boldsymbol{a}^{\prime}=Z^{-T} \boldsymbol{a}$ and $\boldsymbol{\alpha}^{\prime}=Z \boldsymbol{\alpha}$ with $Z \in \mathrm{SL}_{n}(\mathbb{Z})$. Then the Hamiltonian vector fields $X_{a_{k}}=\partial_{\alpha_{k}}$ for 
$k=1, \ldots, n$ of the action variables change consistently $\partial_{\alpha_{k}}=Z^{-T} \partial_{\alpha_{k}}$. The quantum operator associated with the action $a_{k}$ is $\hat{a}_{k}=\mathrm{i} X_{a_{k}}=-\mathrm{i} \partial_{a_{k}}$, as can be easily checked $\hat{a}_{k}=-\mathrm{i}\left(X_{a_{k}}-i_{X_{a_{k}}} \theta\right)+a_{k}=-\mathrm{i} X_{a_{k}}$, since $i_{\partial_{a_{k}}} \sum_{j=1}^{n} a_{j} \mathrm{~d} \alpha_{j}=a_{k}$. Therefore after the change of coordinates the quantum operator takes the form $\boldsymbol{a}^{\prime}=-\mathrm{i} Z^{-T} \partial_{\boldsymbol{\alpha}}$. Then, using Nekhoroshev's idea, if we glue together $r>1$ charts surrounding an isolated singularity of focus-focus type we obtain a non-trivial product $Z=\Pi_{\nu=1}^{r} Z_{\nu}$ of matrices in $\mathrm{SL}_{n}(\mathbb{Z})$. This is equivalent to say that monodromy manifests itself via a non-trivial $\mathrm{SL}_{n}(\mathbb{Z})$-representation $[\gamma] \longmapsto Z=Z([\gamma])$ of the fundamental group $\pi_{1}(A)$ of the base manifold $A$.

Remark 8. We stress the fact that in spectroscopy, monodromy manifests itself precisely through a shift of the energy levels, see e.g. [4] and references therein.

\section{Conclusions and Perspectives}

We have reviewed some general methods to compute the (quantum) monodromy of completely integrable Hamiltonian systems using the geometric quantization procedure. In particular, recovering Weinstein idea, we have detected the monodromy via a choice of the pre-quantum connection and using Nekhoroshev original idea performing a parallel transport along a nontrivial loop around a singularity.

As future work it would be of interest to try to extend our results to fractional monodromy and to study all Duistermaat singularities in the framework of geometric quantization.

\section{Acknowledgements}

I wish to thank to Professor M. Spera for many enlightening discussions on these topics, F. Fassò for many discussion on monodromy and L. Bates for having stimulated me to work on this topic.

\section{References}

[1] Arnold V. and Givental A., Symplectic Geometry. Dynamical Systems IV, Encyclopaedia Math. Sci. vol. 4, Springer, Berlin, 2001, pp 1-138.

[2] Bates L., Monodromy in the Champagne Bottle, ZAMP 42 (1991) 837-847. 
[3] Brylinski J.-L., Loop Spaces, Characteristic Classes and Geometric Quantization, Birkhäuser, Basel, 1993.

[4] Child M., Quantum States in Champagne Bottle, J. Phys. A 31 (1998) 657670.

[5] Cushman R. and Bates L., Global Aspects of Classical Integrable Systems, Birkhäuser, Basel, 1997.

[6] Dazord P. and Delzant T., Le Problème Général des Variables Actions-Angles, J. Diff. Geom. 26 (1987) 223-251.

[7] Duistermaat J., On Global Action-Angle Coordinates, Comm. Pure Appl. Math. 33 (1980) 687-706.

[8] Matveev V., Integrable Hamiltonian Systems with Two Degrees of Freedom. Topological Structure of Saturated Neighborhoods of Saddle-Saddle and Focus Points, Mat. Sb. 187 (1996) 29-58.

[9] Nekhoroshev N., Action-Angle Variables, and Their Generalizations (in Russian), Trudy Moskov. Mat. Obšč. 26 (1972) 181-198.

[10] Vũ Ngoc S., Quantum Monodromy in Integrable Systems, Commun. Math. Phys. 203 (1999) 465-479.

[11] Sansonetto N. and Spera M., Hamiltonian Monodromy Via Geometric Quantization and Theta Functions, J. Geom. Phys. 60 (2010) 501-512.

[12] Sepe D., Topological Classification of Lagrangian Fibrations, J. Geom. Phys. 60 (2010) 341-351.

[13] Weinstein A., Symplectic Manifolds and Their Lagrangian Submanifolds, Adv. Math. 16 (1971) 329-346.

[14] Woodhouse N., Geometric Quantization, Clarendon Press, Oxford, 1992.

[15] Zung N., A Note on Focus-Focus Singularities, Diff. Geom. Appl. 7 (1997) $123-130$.

Nicola Sansonetto

Department of Computer Science

Università di Verona

37134 Verona, ITALY

E-mail address: nicola.sansonetto@gmail.com 